\title{
Thermal Engineering of Metal-Organic Frameworks for Adsorption Applications: A Molecular Simulation Perspective
}

\author{
Jelle Wieme, ${ }^{\dagger}$ Steven Vandenbrande, ${ }^{\dagger}$ Aran Lamaire, ${ }^{\dagger}$ (๑) Venkat Kapil, ${ }^{\ddagger}{ }^{\ddagger}$ Louis Vanduyfhuys, ${ }^{\dagger}$ \\ and Veronique Van Speybroeck* ${ }^{*} \dagger$ \\ ${ }^{\dagger}$ Center for Molecular Modeling, Ghent University, Tech Lane Ghent Science Park Campus A, Technologiepark 46, 9052 \\ Zwijnaarde, Belgium \\ ${ }^{\ddagger}$ Laboratory of Computational Science and Modelling, Institute of Materials, Ecole Polytechnique Fédérale de Lausanne, 1015 \\ Lausanne, Switzerland
}

\section{Supporting Information}

ABSTRACT: Thermal engineering of metal-organic frameworks for adsorption-based applications is very topical in view of their industrial potential, in particular, since heat management and thermal stability have been identified as important obstacles. Hence, a fundamental understanding of the structural and chemical features underpinning their intrinsic thermal properties is highly sought-after. Herein, we investigate the nanoscale behavior of a diverse set of frameworks using molecular simulation techniques and critically compare properties such as thermal conductivity, heat capacity, and thermal expansion with other classes of materials. Furthermore, we propose a hypothetical thermodynamic cycle to estimate the temperature rise associated with adsorption for the most important greenhouse and energy-related gases $\left(\mathrm{CO}_{2}\right.$ and $\left.\mathrm{CH}_{4}\right)$. This macroscopic response on the heat of adsorption connects the intrinsic thermal properties with the adsorption properties and allows us to evaluate their importance.

KEYWORDS: metal-organic frameworks, heat capacity, thermal conductivity, thermal expansion, gas adsorption, molecular simulations, thermal engineering

\section{Adsorption-induced temperature fluctuations}

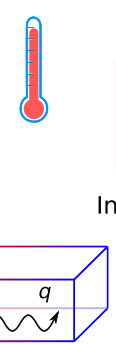

Thermal conductivity

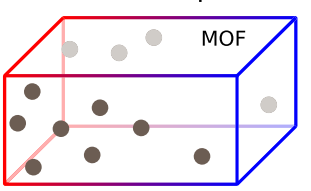

Influence of thermal properties?

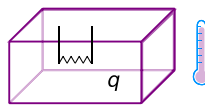

Heat capacity
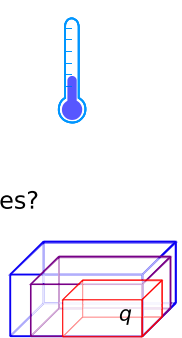

Thermal expansion

\section{INTRODUCTION}

Metal-organic frameworks (MOFs) ${ }^{1}$ belong to the class of the most promising porous materials envisioned to play a role in gas storage and separation. ${ }^{2,3}$ These porous, hybrid inorganicorganic crystalline frameworks possess excellent chemical and physical properties and may, therefore, contribute to solutions for present-day world problems, such as the reduction of the greenhouse effect and the use of sustainable energy. ${ }^{4,5}$ Hence, much fundamental research effort has been devoted to the development of MOFs with a particular emphasis on the improvement of their adsorption properties for carbon capture and methane storage. As MOFs are slowly progressing toward industrial applications, ${ }^{6-9}$ attention increasingly turns toward important thermal engineering issues, such as thermal stability $^{10}$ and heat management. ${ }^{11-13}$ This is particularly relevant as these adsorbents are exposed to large thermal fluctuations, since the adsorption and desorption of guests implies the release or consumption of energy. While efficient heat transport in MOFs will ultimately be limited by their inherent porosity, it is, nevertheless, essential to understand and improve the intrinsic thermal characteristics to reduce the costs of, for instance, compensating heat exchangers. ${ }^{14}$
The released heat of adsorption during charging needs to be dissipated quickly, as an increase in temperature severely diminishes the maximum uptake. In other words, to avoid the system from heating up, the charging rate needs to be constrained. During discharging, a decrease in temperature due to the heat of desorption will increase the number of unusable adsorbates remaining in the pores at the depletion pressure. The amount of gas stored or delivered under realistic dynamical conditions is always lower than that under perfect isothermal conditions. Therefore, for applications based on pressure swing adsorption (PSA), small temperature changes have a detrimental influence on their performance. This bottleneck is often mentioned when considering adsorbed natural gas as an alternative to conventional petroleum-based fuels for transportation vehicles. ${ }^{15-17}$ This problem is not limited to MOFs but extends to other candidate materials, such as activated carbon or zeolites, as well. ${ }^{18,19}$ Nevertheless, for some technologies, it is possible to take advantage of the thermal properties of MOFs. An example is the removal of

Received: July 17, 2019

Accepted: September 26, 2019

Published: September 26, 2019 
(a) Al-Soc-MOF-1

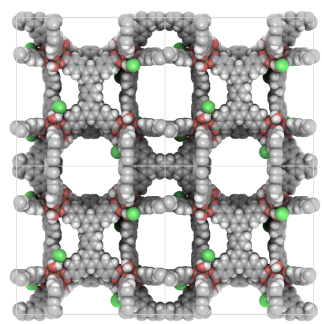

(d) MOF-505

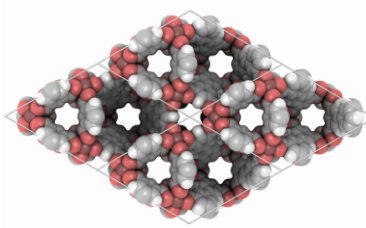

(b) HKUST-1

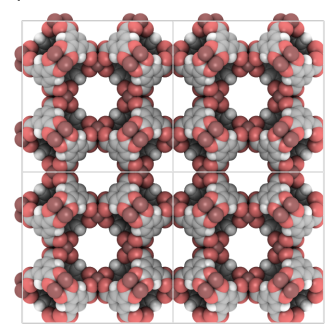

(e) MOF-177

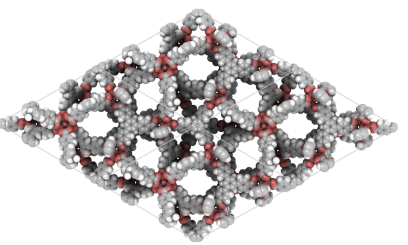

(g) UiO-66

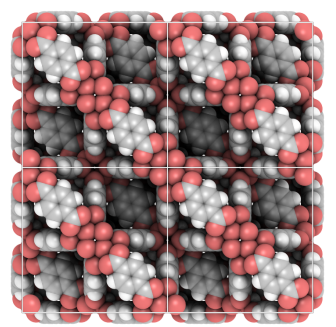

(c) MOF-5

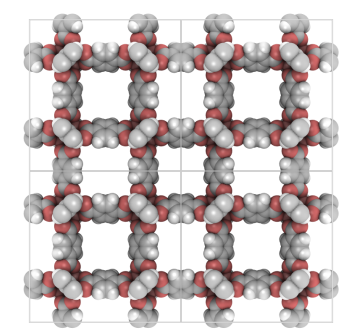

(f) UMCM-1

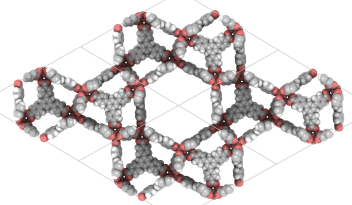

Figure 1. Overview of the MOFs investigated in this study. Al-soc-MOF-1 ${ }^{29}$ is an aluminum-based MOF. HKUST-1 ${ }^{30}$ and MOF-505 ${ }^{31}$ (or NOTT- $\left.100^{32}\right)$ are copper-paddle wheel MOFs. UiO- $66^{33}$ is a zirconium MOF $\left(\mathrm{Zr}_{6} \mathrm{O}_{4}(\mathrm{OH})_{4}\right)$. MOF-5, ${ }^{34} \mathrm{MOF}-177{ }^{35}$ and UMCM-1 $1^{36}$ are $\mathrm{Zn}$ based MOFs $\left(\mathrm{ZnO}_{4}\right)$. The simulated and experimental unit cell parameters of these MOFs are given in the Supporting Information (SI). The figures are generated with iRASPA. ${ }^{37}$

carbon dioxide from flue gas streams using temperature swing adsorption (TSA), ${ }^{20,21}$ where MOFs would have a lower energy penalty to heat to the regeneration temperature than the currently used monoethanolamine technology, as MOFs have a much lower heat capacity. ${ }^{22}$ To compensate for the limited availability of pilot-scale measurements of MOF applications, ${ }^{14,19}$ system performance studies to monitor the thermal effects have started to appear. ${ }^{23,24}$ These studies are mostly based on computational fluid dynamics and require the design of the system (e.g., geometry of the storage tank) together with the adsorbent material's properties. However, there are no systematic studies available mapping simultaneously the various thermal properties, i.e., the thermal conductivity, the heat capacity, and the thermal expansion coefficient, of MOFs. Except for the thermal stability, which is typically measured for every new material, ${ }^{10}$ experimental and even computational data about these properties are mainly limited to the well-known MOFs. This is in sharp contrast to gas adsorption, catalytic, and even mechanical properties. For these properties, large-scale computational screenings of structures have pinpointed important structure-property relations, $^{25,26}$ whereas studies of thermal properties remain limited to toy models ${ }^{13,27}$ or very specific classes of MOFs. ${ }^{28}$

Motivated by this, we evaluated the thermal conductivity, the isobaric specific heat capacity, and the volumetric thermal expansion coefficient from a molecular perspective, by applying molecular simulation techniques to a diverse set of materials representing widely studied classes of rigid MOFs for storage applications (Figure 1). ${ }^{2}$ We included examples with the most important inorganic building blocks, such as the copper-paddle wheel (HKUST-1 ${ }^{30}$ and MOF-505 ${ }^{31}$ ) and $\mathrm{ZnO}_{4}$ (MOF-5, ${ }^{34}$ MOF- $177,{ }^{35}$ and UMCM-1 $\left.{ }^{36}\right)$. Furthermore, we selected UiO$66^{33}$ and Al-soc-MOF- ${ }^{29}$ to represent the $\mathrm{Zr}$-based and $\mathrm{Al}-$ based MOFs, respectively. The set under study includes a number of MOFs for which experimental data on thermal properties have been reported (Table S2). ${ }^{14,38-44}$ To investigate the effect of elongating the organic linkers, we also tested IRMOF-10 and IRMOF-16 as longer linker equivalents of MOF-5 (i.e., IRMOF-1). ${ }^{45}$ The key goal is to obtain insight into how the overall thermal properties are affected by the nanoscale design. In this way, we present the first systematic study of these important properties, giving a broader view on the thermal performance of MOFs and their associated limitations. Additionally, we present a materials property chart, where the thermal properties of MOFs are visualized alongside other classes of materials, such as metals, polymers, and ceramics.

Based on the thermal properties obtained at the molecular level, we, furthermore, propose a thermodynamic cycle to estimate how adsorption impacts the temperature of the adsorbent. This thermodynamic cycle mimics the initial charging process and allows quantifying an upper bound for the intrinsic temperature rise of the system resulting from the release of the heat of adsorption. We investigate the influence of adsorbing the most important greenhouse and energyrelated gases $\left(\mathrm{CO}_{2}\right.$ and $\left.\mathrm{CH}_{4}\right)$ on the material's temperature by applying our protocol on the same set of MOFs, while probing the impact of the intrinsic thermal properties of the MOF on the observed behavior. 


\section{COMPUTATIONAL METHODS}

2.1. Force-Field Derivation. As the computational cost of extracting the thermal properties of MOFs with first-principles molecular dynamics techniques is too high, we instead rely on force-field simulations. Therefore, new system-specific force fields were derived from first-principles cluster data for all MOFs under study. To that end, the quantum mechanical potential energy surface is approximated by a sum of analytic functions of the nuclear coordinates that describe the covalent, electrostatic, and van der Waals interactions. The covalent interactions were fitted using QuickFF, ${ }^{46,47}$ an in-house developed protocol for deriving force fields from first-principles input that has been tested for its adequacy in describing structural, vibrational, mechanical, and thermal properties of MOFs. The underlying idea of QuickFF is to mimic the quantum mechanical potential energy surface in the neighborhood of the equilibrium structure, which is defined by the provided optimized geometry and Hessian, by fitting the unknown parameters featuring in the covalent interactions. The electrostatic interactions were modeled by a Coulomb interaction between Gaussian charge distributions. The van der Waals interactions were described by the MM3-Buckingham model ${ }^{48}$ up to a finite cutoff of $12 \AA$ and were supplemented with tail corrections.

The required first-principles data for the determination of the covalent terms were generated with Gaussian $16^{49}$ using the B3LYP ${ }^{50}$ exchange-correlation functional. The $6-311 G(d, p)$ basis set ${ }^{51}$ was used for the $\mathrm{H}, \mathrm{C}, \mathrm{O}, \mathrm{Al}$, and $\mathrm{Cu}$ atoms, together with the LanL2DZ basis set for $\mathrm{Zn}$ and $\mathrm{Zr} .^{52}$ The atomic charges were derived with the Minimal Basis Iterative Stockholder (MBIS) partitioning scheme ${ }^{53}$ from the all-electron density obtained with Gaussian 16. The MBIS atomic charges of the $\mathrm{Zn}$ - and $\mathrm{Zr}$-clusters were obtained from the $\mathrm{PBE}^{54}$ all-electron density computed with GPAW. ${ }^{55}$ The cluster models for the different MOFs under study are shown in Figures $\mathrm{S} 1-$ S4. A similar approach was followed for $\mathrm{CH}_{4}$ and $\mathrm{CO}_{2}$.

More details on the force-field energy expression, derivation, and validation can be found in SI Section S1. The force fields and input structures are also added to the Supporting Information.

2.2. Molecular Dynamics Simulations. To compute the heat capacity and thermal expansion coefficient, Suzuki-Chin (SC) path integral molecular dynamics (PIMD) simulations ${ }^{56,57}$ were performed at a controlled mechanical pressure of 1 bar and at different temperatures in the range of $100-600 \mathrm{~K}$. PIMD is the method of choice to calculate the equilibrium properties of distinguishable particles with inclusion of nuclear quantum effects at a finite temperature by mapping the quantum partition function onto the classical partition function of an extended system of interacting replicas. ${ }^{58}$ Nevertheless, the inclusion of nuclear quantum effects in molecular dynamics typically comes at a large computational cost. Hence, cost-reduction techniques are required to enable simulations on large systems, such as guest-loaded MOFs. ${ }^{57}$ In this study, we used a combination of multiple-time stepping and ring-polymer contraction to reduce the number of force evaluations ${ }^{59}$ together with the Suzuki-Chin high-order factorization of the quantum partition function to reduce the number of necessary replicas. ${ }^{56}$ The simulations were performed with the universal force engine, $i$ $\mathrm{PI}^{60,61}$ a code that efficiently implements PIMD in the appropriate thermodynamic ensemble. The evaluation of the forces is carried out by other external codes: the covalent interactions are computed with our in-house Yaff code ${ }^{62}$ and the long-range interactions are evaluated with LAMMPS. ${ }^{63}$

These SC PIMD simulations were used to determine the thermal properties, following the procedure of ref 57 . In this advanced PIMD scheme, ${ }^{58}$ all important effects related to the anharmonic and quantum nature of the atomic movements are accounted for, which is necessary to obtain a reliable heat capacity estimate in the presence of guests. ${ }^{5,64}$

Furthermore, the Green-Kubo approach ${ }^{65,66}$ was used to compute the classical thermal conductivity of the empty frameworks. This method provides a relation between a transport coefficient (thermal conductivity) and an integral over a time-correlation function (heat flux) and is based on the fluctuation-dissipation theorem in nonequilibrium statistical mechanics. This formalism yields a method capable of obtaining a transport property from a simulation of a system in thermodynamic equilibrium. In our case, the thermal conductivity is extracted from the heat current autocorrelation function (HCACF). Data used to approximate this property are collected from hundred independent simulations in the classical microcanonical ensemble (NVE) performed with LAMMPS. To obtain the thermal conductivity at room temperature, the system has to be equilibrated first at the desired temperature in the $N V\left(\overline{\bar{h}}_{0}\right) T$ ensemble ${ }^{67}$ before going over to the NVE ensemble. The equilibrium crystallographic unit cell at $300 \mathrm{~K}$ was taken from the SC PIMD simulations. The simulations were performed on super cells to limit finite size effects. ${ }^{68}$ The same approach as that applied on MOF-5 by McGaughey et al. was followed to estimate the thermal conductivity from the HCACF. ${ }^{69,70}$

More details on the molecular dynamics simulations are provided in SI Section S2. A comparison of the simulated thermal properties with experiment is tabulated in Table S2.

2.3. Thermodynamic Cycle. A detailed thermodynamic analysis of our hypothetical cycle is presented in SI Section S3. The main ingredients that need to be computed using molecular simulations are the heat capacity of the guest-loaded structure (see the previous section) and the total heat of adsorption when the empty MOF adsorbs $n$ guest molecules at a temperature $T$. To compute the latter, we note that this can be written as (SI Section S3)

$$
\Delta H_{1 \mathrm{~b}}=\Delta U^{S}(0 \rightarrow n)+P \Delta V^{S}(0 \rightarrow n)-n\left(U_{T}^{R}(1)+k_{\mathrm{B}} T\right)
$$

The first term in this expression, i.e., $\Delta U^{S}(0 \rightarrow n)$, is the change in internal energy of the thermodynamic system $S$, which corresponds to the difference in energy between the guest-loaded MOF and the empty MOF under isothermal-isobaric conditions. The second term is typically rather small and is associated with the mechanical work determined by the product of the controlled mechanical pressure $P$ and the change in volume $\Delta V$ of the system when adsorbing $n$ guest molecules. Both terms can straightforwardly be extracted from the SC PIMD simulations required for the heat capacity of the empty and guest-loaded MOF. The last term coincides with the enthalpy change of an ideal gas reservoir $R$, from which $n$ gas molecules are removed. The internal energy $U_{T}^{R}(1)$ can be found by performing a SC PIMD simulation on a single gas molecule at a temperature $T$.

Four different loadings of $\mathrm{CH}_{4}$ and $\mathrm{CO}_{2}$ are considered to enable a consistent comparison between the different MOFs, namely, 20, 50, 75 , and $100 \%$ of the equilibrium loading at $300 \mathrm{~K}$ and 100 bar. This high-pressure loading was computed using Grand Canonical Monte Carlo (GCMC) simulations with RASPA. ${ }^{71}$ Snapshots from these GCMC simulations were subsequently used in the SC PIMD simulations of the guest-loaded MOFs. More details on the GCMC simulations and a comparison with experimental isotherms can be found in SI Section S3.2.

\section{RESULTS AND DISCUSSION}

3.1. Thermal Properties of MOF Adsorbents. Before comparing the properties of MOFs with other classes of materials, such as metals, polymers, and ceramics, the three investigated thermal properties are discussed separately for our diverse set of MOFs.

3.1.1. Thermal Conductivity. Heat dissipation and efficient thermal transport are big issues in the context of storage applications with charge and discharge cycles. ${ }^{2,73}$ Hence, the low thermal conductivity of MOFs is perceived as a fundamental problem of the materials. The main origins of the bad thermal conductance in MOFs are known and result from the intrinsic chemical diversity and porosity. ${ }^{13,74,75}$ Chemical diversity or heterogeneity introduces a mismatch between atomic masses and bond strengths, which results in phonon scattering. Moreover, the large empty voids hinder the 
Table 1. Structural and Thermal Properties of a Number of MOFs ${ }^{a}$

\begin{tabular}{|c|c|c|c|c|c|c|c|c|c|}
\hline material & Al-soc-MOF-1 & HKUST-1 & MOF-505 & $\mathrm{UiO}-66$ & MOF-5 & IRMOF-10 & IRMOF-16 & UMCM-1 & MOF-177 \\
\hline$\rho\left(\mathrm{kg} \mathrm{m}^{-3}\right)$ & 343 & 851 & 888 & 1162 & 555 & 304 & 193 & 366 & 406 \\
\hline $\operatorname{ASA}\left(\mathrm{m}^{2} \mathrm{~g}^{-1}\right)$ & 5016 & 2229 & 2423 & 1246 & 3894 & 5230 & 6123 & 4584 & 4899 \\
\hline void fraction (\%) & 80 & 66 & 64 & 47 & 77 & 86 & 90 & 83 & 80 \\
\hline$\kappa\left(\mathrm{W} \mathrm{m}^{-1} \mathrm{~K}^{-1}\right)$ & 0.22 & 0.45 & $1.16-1.26$ & 0.87 & 0.29 & 0.09 & 0.07 & $0.07-0.13$ & $0.08-0.09$ \\
\hline$C_{\mathrm{P}}\left(\mathrm{J} \mathrm{kg}^{-1} \mathrm{~K}^{-1}\right)$ & 1051 & 774 & 800 & 758 & 786 & 860 & 913 & 865 & 894 \\
\hline$\beta_{\mathrm{V}}\left(10^{-6} \mathrm{~K}^{-1}\right)$ & -19 & -15 & -17 & -9 & -41 & -48 & -52 & -47 & -39 \\
\hline
\end{tabular}

${ }^{a_{T}}$ The structural properties (density $\rho$, accessible surface area (ASA), and probe-occupiable void fraction) were derived from the optimal structure at $0 \mathrm{~K}$ using Zeo++ ${ }^{72}\left(\mathrm{~N}_{2}\right.$ probe molecule with kinetic diameter $\left.3.64 \AA\right)$. The thermal properties are simulated at room temperature $(300 \mathrm{~K})$ : thermal conductivity $\kappa$, isobaric heat capacity $C_{\mathrm{P}}$, and volumetric thermal expansion coefficient $\beta_{\mathrm{V}}$. A comparison of the simulated thermal properties with experiments is tabulated in Table S2.

efficient propagation of phonons through the porous crystal structures. While a high thermal conductivity for these materials might, therefore, not be realistic, it should be possible to identify structural and chemical descriptors that describe the best-performing frameworks.

The thermal conductivity is often defined through the empirical Fourier's law for heat conduction

$$
\bar{q}=-\overline{\bar{\kappa}} \cdot \bar{\nabla} T
$$

where $\bar{q}$ is the local heat flux density, $\bar{\nabla} T$ is the local temperature gradient, and $\overline{\bar{\kappa}}$ is the second-order thermal conductivity tensor. The thermal conductivity coefficient can depend on the crystal direction in MOFs, ${ }^{13}$ but most structures in our set are isotropic and can be described by one thermal conductivity coefficient (Table 1). For MOF-505, UMCM-1, and MOF-177, we have indicated the limited spread on the coefficients due to the anisotropic cell directions in Table 1 .

Within the class of MOFs, there are some important trends to be noted in Figure 2 regarding $\kappa$. First, to systematically investigate the effect of elongating the linkers or increasing the size of the pores, IRMOF-10 and IRMOF-16 were compared with MOF-5 (blue dotted line). As the low thermal conductivity of MOFs mainly stems from the large empty voids, it is not a surprise that the coefficient of heat conduction decreases with increasing linker length. Indeed, it goes down

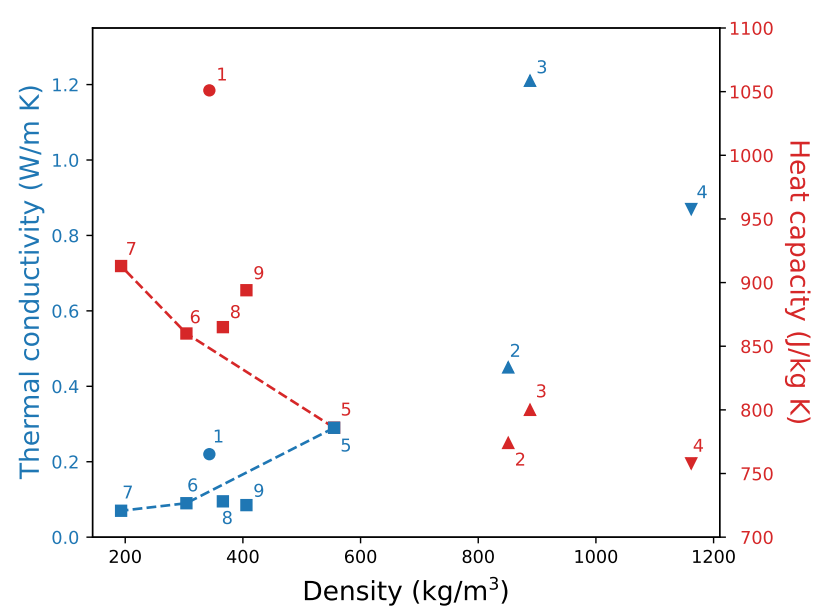

Figure 2. Thermal conductivity (blue) and heat capacity (red) as a function of the density at room temperature. The markers indicate the inorganic node: $\mathrm{Zr}_{6} \mathrm{O}_{8} \mathrm{H}_{4}$ (down-triangle), $\mathrm{ZnO}_{4}$ (square), $\mathrm{CuC}_{4} \mathrm{O}_{8}$ (up-triangle), and $\mathrm{Al}$ (circle). The IRMOF-series (MOF-5, IRMOF10, and IRMOF-16) are connected by a line. $1=$ Al-soc-MOF-1, $2=$ HKUST-1, 3 = MOF-505, 4 = UiO-66, 5 = MOF-5, 6 = IRMOF-10, 7 $=$ IRMOF-16, 8 = UMCM-1, and 9 = MOF-177. from MOF-5 toward IRMOF-16, in line with the toy model predictions of Babaei et al. ${ }^{13}$

The suggested correlation between the size of the pores and the thermal conductivity appears to be relatively independent of the underlying chemical structure. MOFs with a density below $500 \mathrm{~kg} \mathrm{~m}^{-3}$ have ultralow thermal conductivities. Unfortunately, these are the most porous frameworks, which can store the largest amount of gas at high pressure (see textural properties in Table 1). Moreover, MOF-5, HKUST-1, and $\mathrm{UiO}-66$ have a similar linker length in between the inorganic nodes, but their performance seems to be solely dominated by the density. MOF-505 with copper-paddle wheels in the popular NbO-topology has a significantly higher value than the other frameworks and could be an interesting topology with improved thermal characteristics to start from.

3.1.2. Specific Heat Capacity. Another thermal property that has been recognized to influence the actual performance of adsorption-based technologies is the heat capacity. As mentioned in the Introduction, for applications based on TSA, a low value is desired as the adsorbent heat capacity largely affects the energy penalty to heat the system to the regeneration temperature. In contrast, for PSA processes, a high adsorbent heat capacity is beneficial, as it limits the temperature peaks during charging and discharging. ${ }^{15,76}$

The specific heat capacity $\left(\mathrm{J} \mathrm{kg}^{-1} \mathrm{~K}^{-1}\right)$ describes the amount of energy required to increase the material's temperature and is defined as

$$
C_{\mathrm{P}}=\frac{1}{M}\left(\frac{\partial H}{\partial T}\right)_{P}
$$

where $H$ is the molar enthalpy of the system and $M$ is the molar mass. It has previously been measured for a selected number of MOFs, ${ }^{14,39,40}$ and these reported values suggested a specific heat capacity in between 700 and $1000 \mathrm{~J} \mathrm{~kg}^{-1} \mathrm{~K}^{-1}$ at room temperature. ${ }^{77}$ It is sensitive to the temperature and can change by more than $65 \%$ in the interval between 200 and 400 $\mathrm{K}^{57}$

The results in Table 1 are evidently in line with the proposed experimental range (and with the available experimental results (Figure S5)). Figure 2 shows that MOFs possessing the lowest density, i.e., Al-soc-MOF-1, MOF-177, and UMCM-1, have a larger specific heat capacity. This is also reflected in the IRMOF-series. The inverse correlation between specific heat capacity on a mass basis and the density is also found in regular solids (Figure S13). A different picture emerges when considering the volumetric heat capacity $\left(=\rho C_{\mathrm{P}}\right.$, Figure $\left.\mathrm{S} 7\right)$, where the most porous frameworks possess the lowest value and vice versa. 
The aluminum-based Al-soc-MOF-1 has the highest value per gram MOF, which is due to the lighter inorganic node, as compared to zinc, copper, and zirconium, and the presence of a tetratopic linker containing many light atoms. This can be understood by realizing that the specific heat capacity is a measure for the available degrees of freedom on a mass basis.

3.1.3. Thermal Expansion. A third thermal property that needs to be dealt with in applications is the thermal expansion behavior. This is characterized by a volumetric thermal expansion coefficient $\beta_{\mathrm{V}}$

$$
\beta_{\mathrm{V}}=\frac{1}{V}\left(\frac{\partial V}{\partial T}\right)_{P}
$$

The difference between the thermal expansion coefficients of the materials in contact with the adsorbent bed may give rise to mechanical failure. This thermal expansion mismatch is an important issue that has been mentioned before, mainly in the context of thin-film-based applications of MOFs. ${ }^{78}$ MOFs such as MOF-5 and HKUST-1 even shrink with increasing temperature as opposed to most other materials possessing a positive thermal expansion. ${ }^{41,42}$ However, the studied MOFs (see Table 1) and an increasing number of reports ${ }^{79}$ suggest that negative thermal expansion is not the exception but might be quite common for MOFs. MOFs containing the $\mathrm{ZnO}_{4}$ inorganic node have notably larger negative thermal expansion coefficients, whereas UiO-66 exhibits a smaller coefficient that might be explained by the higher connectivity of the inorganic nodes. The origin of negative thermal expansion has been investigated for several of these structures (MOF-5, IRMOF10, IRMOF-16, HKUST-1, and UiO-66). ${ }^{28,41,42,80,81}$ These studies have suggested that low-energy transverse vibrational modes play an important role, and that it strongly depends on the topology of the structure. ${ }^{79}$

3.1.4. Comparison with Other Materials. To put the obtained values for the different properties into perspective, a comparison is made with more common classes of materials, such as metals, ceramics, and polymers. ${ }^{82}$ This is done in the materials property chart in Figure 3, where a two-dimensional representation based on the simulated specific heat capacity and thermal conductivity is shown for the MOFs under study alongside experimental data for other materials (see SI Section

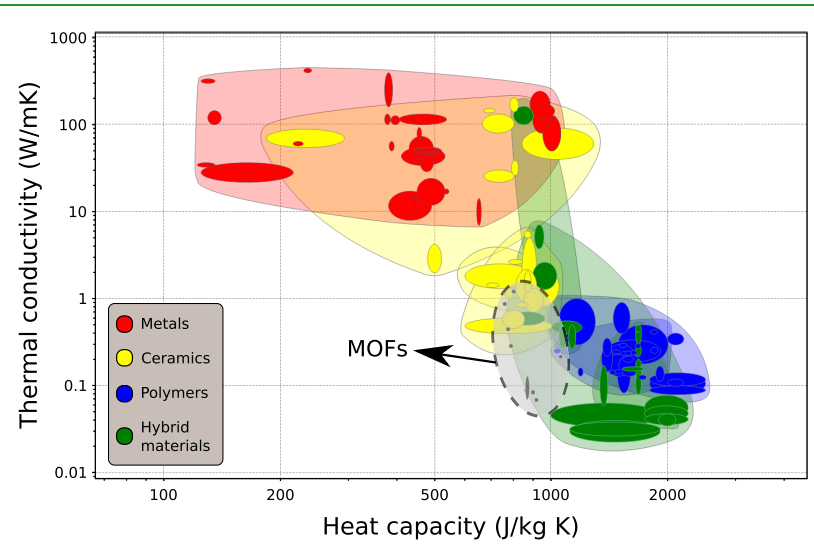

Figure 3. Materials property chart displaying the thermal conductivity and the specific heat capacity of MOFs alongside other classes of materials, namely, metals (and alloys), ceramics (glasses and (non-) technical ceramics), polymers, and hybrid materials (composites, foams, and natural materials). An overview of the used materials is given in the SI.
S4). It should be noted that our simulated results contain intrinsic model uncertainties and that they might quantitatively differ from experimental measurements, which have not been available for most MOFs to date. However, in this work, we will mainly focus on qualitative trends. As such, the data undeniably show that MOFs have thermal properties at the crossing of the ceramics, polymers, and hybrid materials (natural materials and composites). More specifically, the MOFs with the lowest thermal conductivity are relatively unique in this set of materials. The ones with the highest value lie in the neighborhood of typical ceramics, such as borosilicate glass $\left(\kappa \approx 1-1.3 \mathrm{~W} \mathrm{~m}^{-1} \mathrm{~K}^{-1}, C_{\mathrm{P}} \approx 800 \mathrm{~J} \mathrm{~kg}^{-1} \mathrm{~K}^{-1}\right)$ and brick $\left(\kappa \approx 0.45-0.75 \mathrm{~W} \mathrm{~m}^{-1} \mathrm{~K}^{-1}, C_{\mathrm{P}} \approx 800 \mathrm{~J} \mathrm{~kg}^{-1} \mathrm{~K}^{-1}\right)$. Finally, Alsoc-MOF-1 compares relatively well with Teflon.

In the Supporting Information, the separate thermal properties are displayed as a function of the density in a comparison with the same materials (Figures S12-S14). This reveals, for instance, that MOFs provide a heat conduction similar to that of natural materials (e.g., softwood) and specific heat capacities on the order of those of foams (e.g., ceramic foam) for the same density. The thermal expansion behavior is rather exceptional. All other material representatives have positive thermal expansion coefficients, whereas the opposite is true for the investigated MOFs. However, the magnitude of the thermal expansion coefficient is quite similar to that of foams. One might envisage the production of composite materials, which behave as zero thermal expansion materials.

Also note that we evaluated all properties on infinite perfect single crystals, whereas in reality, MOFs are loose powders that will have to be shaped using compaction methods. ${ }^{83,84}$ In principle, the single-crystal thermal conductivity should represent an upper limit for the material and should, therefore, be higher than that of the MOF in powder form. Densification of these MOF powders will, in some cases, improve the heat transfer characteristics of the sample (for example, due to plastic deformation), even though the intrinsic properties of the single-crystal material are not changed. ${ }^{14}$ In addition, making MOF composites with expanded natural graphite together with compaction was already shown to enhance the thermal properties of the system. ${ }^{85-87}$ To succeed in system integration of MOF powders together with an acceptable thermal performance, engineering approaches beyond improving the intrinsic MOF thermal properties, such as heat exchangers or MOF composites, will have to be developed. $^{88-90}$

Furthermore, up to now we have focused on the intrinsic thermal properties of the adsorbent in the absence of guests. Of course, in practical applications the pores will often be filled with adsorbates. How this influences all three thermal properties will be the topic of a future study. On the one hand, for the thermal conductivity and the heat capacity, a limited number of isolated case studies are already available. ${ }^{57,73,75,91}$ They show that the influence can be quite drastic, and more studies are required to explain the observed phenomena. On the other hand, guests have been used to tune the thermal expansion behavior of MOFs, ${ }^{92,93}$ which is not limited to the flexible MOFs that display phase transitions and massive volume changes under guest adsorption. ${ }^{94,95}$

3.2. Thermodynamic Cycle for Adsorption. So far, a clear overview has been obtained for the thermal properties of several MOFs. However, to use the materials in practical applications, this knowledge should be coupled with the adsorption properties of guests. Herein, we focus on the heat 
generated when gas molecules adsorb into the pores of the material. To this end, a hypothetical thermodynamic cycle is presented, which allows predicting the temperature rise within a MOF due to guest adsorption. This quantity depends not only on the intrinsic thermal properties of the adsorbent but also on the guest-host and guest-guest interactions of the adsorbates. This hybrid, computational descriptor could be used in future screenings to probe the thermal performance of a framework.

This thermodynamic cycle starts from an empty MOF at temperature $T$ and ends at a guest-loaded final state at a higher temperature $T+\Delta T$. We consider an idealized scenario in which the framework instantaneously adsorbs the guest molecule as well as instantaneously absorbs the resulting heat of adsorption. During the actual filling process, a complex dynamical combination of mass and thermal transport takes place at the molecular level, which was nicely divided into different phenomena by Babaei et al. ${ }^{96}$ We do not include interface effects but investigate how the bulk material will thermally respond to the adsorption of guest molecules. At the system's level (i.e., including equipment different than the adsorbent), other effects such as compression work and thermal mass from the inlet can play a role. ${ }^{90}$ Below, we describe our idealized process and discuss its assumptions. In the Supporting Information (SI Section S3), we present a detailed thermodynamic analysis of our hypothetical cycle.

Figure 4 displays the different steps and thermodynamic states of the thermodynamic cycle. The initial state (the empty MOF adsorbent) adsorbs $n$ guest molecules (1), a step which

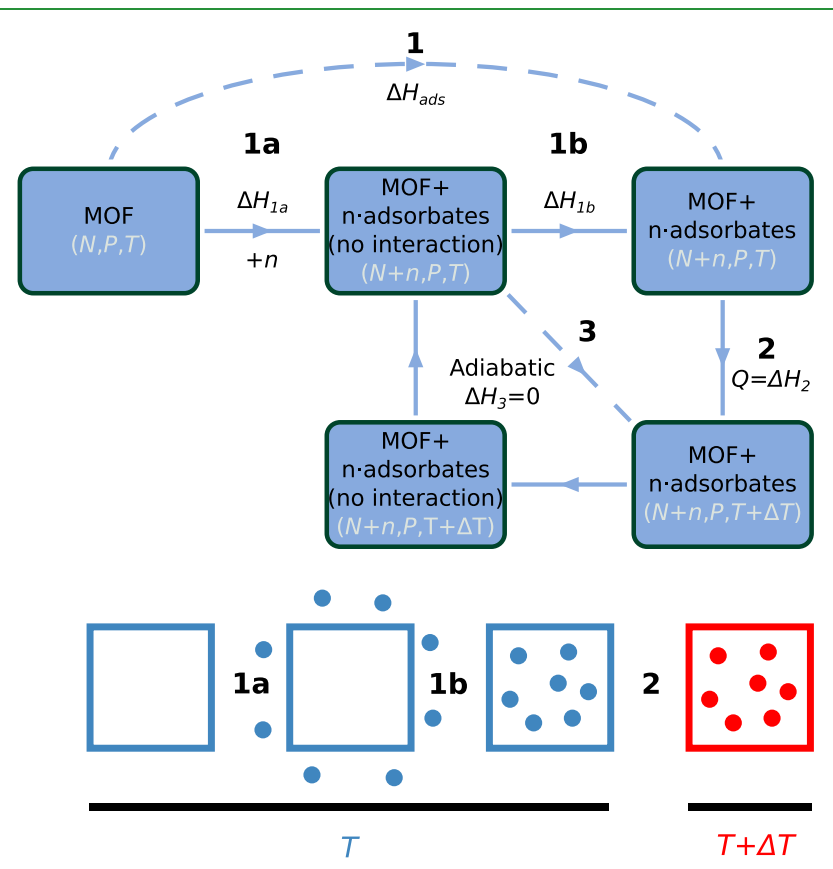

Figure 4. Definition of our hypothetical thermodynamic cycle. After step 1, $n$ guest molecules are adsorbed in the MOF ( $N$ atoms) under isothermal $(T)$ and isobaric $(P)$ conditions. This step is subdivided into two parts. First $(\mathbf{1 a}), n$ noninteracting particles are introduced in the MOF. Subsequently, these guests are physisorbed in $\mathbf{1 b}$. During step 2, the released heat of adsorption is used to heat up the thermodynamic system to a temperature $T+\Delta T$. We assume that $\mathbf{1 b}$ $+2=3$ proceeds adiabatically. Molecular simulations are performed to obtain the relevant quantities. evolves under isothermal and isobaric conditions and is subdivided into

- 1a: the introduction of $n$ noninteracting molecules in the MOF, i.e., neither guest-guest nor guest-host interactions are already present.

- 1b: the physisorption of $n$ particles (including all physical interactions).

After step 1a, the thermodynamic system $S$ is thermally isolated and does not allow for mass and heat transfer with the gas reservoir. The internal energy, $U$, can still change due to the exchange of $P \Delta V$ work. The heat, $Q$ required during step 2 to increase the temperature with $\Delta T$ can be found by integrating the isobaric heat capacity of the guest-loaded MOF from $T$ to $T+\Delta T$.

We consider an adiabatic process $3(=\mathbf{1 b}+\mathbf{2})$, which enables us to calculate $\Delta T$. Based on the first law of thermodynamics, we can write the following integral equation for the unknown $\Delta T$

$$
-\Delta H_{1 b}=\Delta H_{2}=Q=\int_{T}^{T+\Delta T} C_{P}(\mathcal{T} ; n) \mathrm{d} \mathcal{T}
$$

This equation emphasizes the importance of the heat capacity of the adsorbent including adsorbates $C_{p}(\mathcal{T} ; n) .{ }^{57}$ As discussed in the Computational Methods Section, all relevant quantities can be extracted from SC PIMD simulations.

In other words, the heat of adsorption released due to the stabilizing guest-host and guest-guest interactions is subsequently used to heat up the thermodynamic system and to increase the temperature during step 2 . We assume adiabatic walls, as this process happens very rapidly, with fast temperature rises. ${ }^{19}$ This assumption is supported by a molecular simulation study by Babaei et al. ${ }^{96}$ They showed that the conduction of the heat generated due to adsorption is faster than the diffusion of the guest molecules, making the temperature spatially uniform at any time. The heat can, however, not easily be transferred to the pure gas region in contact with the MOF interface due to the high thermal resistance at this interface. They concluded that the interface presents a bottleneck, making the heat transfer to the surroundings a slow process. Similarly, Beckner and Dailly experimentally observed, in a pilot-scale study for vehicular storage, temperature peaks of $30 \mathrm{~K}$ when adsorbing methane at 40 bar and a temperature which only equilibrated after 2 h. ${ }^{19}$

The assumption of an adiabatic process presents an ideal limiting case, which will result in an upper bound for a temperature rise at the material's level. The other extreme case is the isothermal process, where the heat is immediately dissipated through its surroundings. In practice, heat exchangers in contact with the adsorbent bed are used to steer the process toward the latter, as this results in a more efficient (dis)charging process.

We display the most important results for our thermodynamic cycle in Figure 5. This graph indicates the increasing temperature $(T+\Delta T)$ as a function of loading for our set of materials based on guest-loaded MOF simulations. We find a temperature rise $\Delta T$ ranging from 100 to $250 \mathrm{~K}$ depending on the framework and the adsorbate at a high-pressure loading of 100 bar. Figure 5 shows that $\mathrm{CH}_{4}$ has less influence on the material's temperature than $\mathrm{CO}_{2}$. It should be noted that more $\mathrm{CO}_{2}$ molecules are adsorbed than $\mathrm{CH}_{4}$ at the same gas pressure (Table S5). The copper-paddle wheel MOFs, HKUST-1 and MOF-505, have the highest increase for 


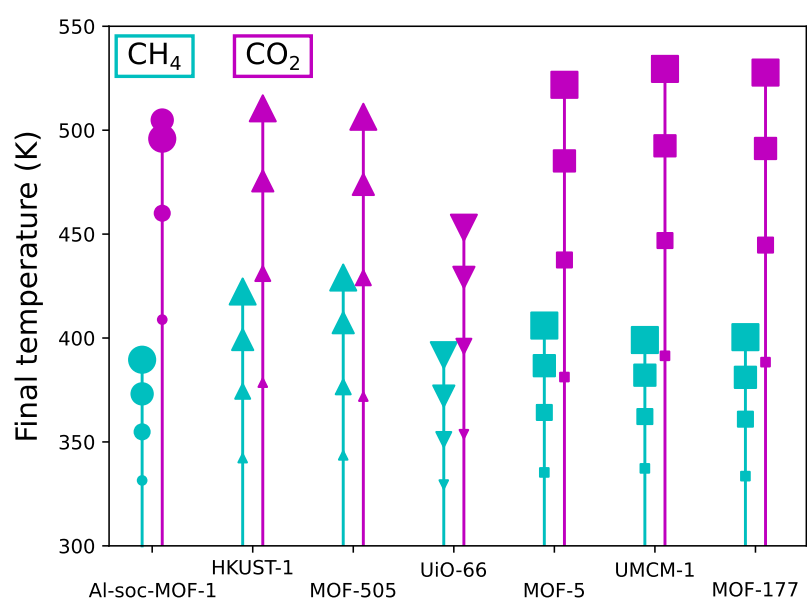

Figure 5. Temperature rise of MOFs when adsorbing $\mathrm{CH}_{4}$ (cyan) and $\mathrm{CO}_{2}$ (magenta) at $300 \mathrm{~K}$. The size of the symbols increases with the loading and represents $25,50,75$, and $100 \%$ of the loading at $300 \mathrm{~K}$ and 100 bar. The markers indicate the inorganic node: $\mathrm{Zr}_{6} \mathrm{O}_{8} \mathrm{H}_{4}$ (down-triangle), $\mathrm{ZnO}_{4}$ (square), $\mathrm{CuC}_{4} \mathrm{O}_{8}$ (up-triangle), and $\mathrm{Al}$ (circle). The numerical results of these data are presented in Table S5.

methane, whereas the Zn-MOFs (MOF-5, UMCM-1, and MOF-177) respond the most to $\mathrm{CO}_{2}$. UiO-66 has the lowest increase in temperature for both adsorbates. These trends are already visible at lower loadings (25\% of the high-pressure loading), and $\Delta T$ in general keeps increasing with loading. Although this temperature increase is an idealized upper bound, its magnitude is indicative of the huge temperature fluctuations that are associated with gas adsorption. For some MOFs, it is even on the same order as their decomposition temperature (e.g., $550 \mathrm{~K}$ for HKUST- $1^{39}$ ) in the case of 100 bar $\mathrm{CO}_{2}$ adsorption.

Now it becomes possible to assess whether improving the heat capacity is a viable and realistic strategy to reduce the temperature peaks during (dis)charging. Figure 6 displays the correlation between the heat of adsorption $\left(-\Delta H_{1 \mathrm{~b}}\right.$ ) (on a mass basis) and the temperature increase $\Delta T$ of the

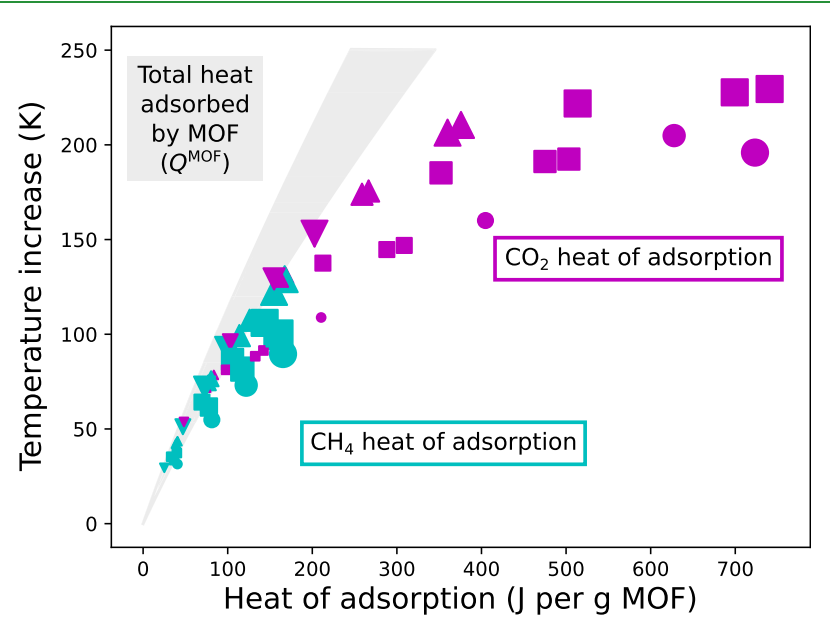

Figure 6. Temperature increase (starting from $300 \mathrm{~K}$ ) after adsorbing $\mathrm{CH}_{4}$ and $\mathrm{CO}_{2}$ as a function of the heat of adsorption. The size of the symbols increases with the loading and represents $25,50,75$, and $100 \%$ of the loading at $300 \mathrm{~K}$ and 100 bar. The markers indicate the inorganic node: $\mathrm{Zr}_{6} \mathrm{O}_{8} \mathrm{H}_{4}$ (down-triangle), $\mathrm{ZnO}_{4}$ (square), $\mathrm{CuC}_{4} \mathrm{O}_{8}$ (up-triangle), and $\mathrm{Al}$ (circle). The gray shaded area indicates the total heat that can be adsorbed by the MOF. thermodynamic system containing adsorbent and adsorbates. The symbol size and shape refer to the number of adsorbates and the type of framework, respectively. It comes as no surprise that the temperature rise increases with increasing heat of adsorption. Furthermore, as methane has weaker guest-guest and guest-host interactions than $\mathrm{CO}_{2}$, the released heat is lower and so is $\Delta T$, as observed above.

To understand how much heat the framework atoms can absorb, we focus here on the heat capacity of the empty framework. The gray shaded area covers the specific heat $Q^{\mathrm{MOF}}$ that the MOFs under study can intrinsically absorb (based on the empty framework simulations) according to the following relation:

$$
Q^{\mathrm{MOF}}(\Delta T)=\int_{300}^{300+\Delta T} C_{\mathrm{P}}^{\mathrm{MOF}}(\mathcal{T}) \mathrm{d} \mathcal{T}
$$

with $Q^{\mathrm{MOF}}$ on the horizontal axis and $\Delta T$ on the vertical axis of Figure 6. This is a good approximation, as the presence of guests does not significantly modify the contribution of the MOF to the heat capacity. ${ }^{57}$ Hence, the horizontal distance between the gray area and the simulated results illustrates how much heat of adsorption is captured by the adsorbates in the pores. For most loadings of $\mathrm{CO}_{2}$, this is significantly more than the heat absorbed by the framework itself. The mixture of guests inside the pores, thus, dominates the total heat capacity and, thus, controls the temperature rise. The narrow range of observed heat capacities for MOFs does not offer much potential in limiting temperature spikes by optimizing within the space of known MOFs. Interesting is also the apparent plateau in $\Delta T$ for the heat of adsorption in a wide range between 500 and almost $800 \mathrm{~J}$ per gram MOF. This suggests that the additional heat of adsorption beyond $500 \mathrm{~J} \mathrm{~g}^{-1}$ MOF can be stored rather efficiently by the inter- and intramolecular interactions of the adsorbates.

Nevertheless, it is clear that for weaker interacting molecules, such as methane, most heat can be adsorbed by the framework. This can be understood by the fact that the gray shaded area is close to the simulated methane-loaded data. However, in this region, the narrow spread on the performance of the diverse set of MOFs again suggests that selecting a MOF with a higher heat capacity within the currently known range will only have a minor impact on the temperature peaks. This will probably also be the case when the adsorbent is coupled with a heat exchanger.

Overall, the analysis thus indicates that replacing a MOF by another MOF with similar adsorption properties but with a higher heat capacity in the studied range will not significantly modify the performance. Rather, new frameworks should be constructed to extend the width of the gray area toward the right, such that the materials are capable of absorbing more heat. As we showed that MOFs are relatively clustered in the materials property chart for thermal properties, conceptually new structures should be devised.

\section{CONCLUSIONS}

MOFs have been recognized as promising adsorbents. It has been envisioned that these materials will play a key role in adsorption-based technologies such as natural gas storage. However, an actual system is also subjected to thermal effects, as adsorption and desorption processes involve the release or consumption of energy. This will result in large temperature changes that have a detrimental effect on the desired 
performance. Engineering strategies exist that can reduce these effects; however, so far it has been unclear as to what extent the intrinsic thermal properties play a role in the thermal effects of the overall process.

In this work, we assessed the thermal conductivity, heat capacity, and thermal expansion of a diverse set of MOFs using molecular simulation methods and indicated how important characteristics such as the density and the chemical nature of their inorganic building blocks influence them. We showed that the thermal conductivity and heat capacity of MOFs are generally low in comparison to other standard materials and that it will be difficult to fundamentally improve the material's properties. The negative thermal expansion behavior of MOFs has already been reported for various structures and was found here as well for a diverse set.

We used the knowledge of these intrinsic thermal properties to estimate as to what extent the temperature of the material would rise when adsorbing $\mathrm{CH}_{4}$ and $\mathrm{CO}_{2}$. To this end, a hypothetical thermodynamic cycle was proposed that relies on macroscopic quantities found through simulations at the molecular level. Our thermodynamic considerations reveal significant temperature increases at the material's level. This quantity itself is a relevant materials parameter balancing both thermal and adsorption properties in MOFs. In this way, it was clear that the adsorption of $\mathrm{CO}_{2}$ has much more impact on the temperature than that of the weaker interacting $\mathrm{CH}_{4}$. Furthermore, by visualizing the correlation between the increase in temperature and the heat of adsorption, we were able to assess the relevance of the heat capacity of the framework and note its limited impact. Our simulations suggest that selecting a MOF with a higher heat capacity would only be a viable strategy if new and improved MOFs are synthesized.

\section{ASSOCIATED CONTENT}

\section{S Supporting Information}

The Supporting Information is available free of charge on the ACS Publications website at DOI: 10.1021/acsami.9b12533.

Force-field parameters and input structures are added separately (ZIP)

More details on the force-field energy expression, derivation, and validation (Section S1); methods used to calculate the thermal properties (Section S2); thermodynamic cycle (Section S3) (PDF)

\section{AUTHOR INFORMATION}

\section{Corresponding Author}

*E-mail: Veronique.VanSpeybroeck@UGent.be.

\section{ORCID}

Jelle Wieme: 0000-0002-4841-2608

Aran Lamaire: 0000-0003-0093-5490

Venkat Kapil: 0000-0003-0324-2198

Veronique Van Speybroeck: 0000-0003-2206-178X

\section{Notes}

The authors declare no competing financial interest.

\section{ACKNOWLEDGMENTS}

This work is supported by the Fund for Scientific Research Flanders (FWO). V.V.S. acknowledges funding from the European Union's Horizon 2020 research and innovation program (consolidator ERC grant agreement No. 647755-
DYNPOR (2015-2020)). This work is furthermore supported by the Research Board of Ghent University (BOF). The computational resources and services used in this work were provided by VSC (Flemish Supercomputer Center), funded by Ghent University, FWO, and the Flemish Government department EWI. V.K. acknowledges financial support by the Swiss National Science Foundation (project ID 200021159896).

\section{REFERENCES}

(1) Furukawa, H.; Cordova, K. E.; O’Keeffe, M.; Yaghi, O. M. The Chemistry and Applications of Metal-Organic Frameworks. Science 2013, 341, No. 1230444.

(2) He, Y.; Chen, F.; Li, B.; Qian, G.; Zhou, W.; Chen, B. Porous Metal-Organic Frameworks for Fuel Storage. Coord. Chem. Rev. 2017, 373, 167-198.

(3) Li, H.; Wang, K.; Sun, Y.; Lollar, C. T.; Li, J.; Zhou, H.-C. Recent Advances in Gas Storage and Separation Using Metal-Organic Frameworks. Mater. Today 2018, 21, 108-121.

(4) Boot-Handford, M. E.; Abanades, J. C.; Anthony, E. J.; Blunt, M. J.; Brandani, S.; Mac Dowell, N.; Fernández, J. R.; Ferrari, M.-C.; Gross, R.; Hallett, J. P.; Haszeldine, R. S.; Heptonstall, P.; Lyngfelt, A.; Makuch, Z.; Mangano, E.; Porter, R. T. J.; Pourkashanian, M.; Rochelle, G. T.; Shah, N.; Yao, J. G.; Fennell, P. S. Carbon Capture and Storage Update. Energy Environ. Sci. 2014, 7, 130-189.

(5) Chu, S.; Cui, Y.; Liu, N. The Path Towards Sustainable Energy. Nat. Mater. 2017, 16, 16-22.

(6) Silva, P.; Vilela, S. M. F.; Tomé, J. P. C.; Almeida Paz, F. A. Multifunctional Metal-Organic Frameworks: From Academia to Industrial Applications. Chem. Soc. Rev. 2015, 44, 6774-6803.

(7) Beckner, M.; Dailly, A. Adsorbed Methane Storage for Vehicular Applications. Appl. Energy 2015, 149, 69-74.

(8) Rubio-Martinez, M.; Avci-Camur, C.; Thornton, A. W.; Imaz, I.; Maspoch, D.; Hill, M. R. New Synthetic Routes Towards MOF Production at Scale. Chem. Soc. Rev. 2017, 46, 3453-3480.

(9) DeSantis, D.; Mason, J. A.; James, B. D.; Houchins, C.; Long, J. R.; Veenstra, M. Techno-economic Analysis of Metal-Organic Frameworks for Hydrogen and Natural Gas Storage. Energy Fuels 2017, 31, 2024-2032.

(10) Howarth, A. J.; Liu, Y.; Li, P.; Li, Z.; Wang, T. C.; Hupp, J. T.; Farha, O. K. Chemical, Thermal and Mechanical Stabilities of MetalOrganic Frameworks. Nat. Rev. Mater. 2016, 1, No. 15018.

(11) de Lange, M. F.; Ottevanger, C. P.; Wiegman, M.; Vlugt, T. J. H.; Kapteijn, F. Crystals for Sustainability - Structuring Al-based MOFs for the Allocation of Heat and Cold. CrystEngComm 2015, 17, 281-285.

(12) Mason, J. A.; Oktawiec, J.; Taylor, M. K.; Hudson, M. R.; Rodriguez, J.; Bachman, J. E.; Gonzalez, M. I.; Cervellino, A.; Guagliardi, A.; Brown, C. M.; Llewellyn, P. L.; Masciocchi, N.; Long, J. R. Methane Storage in Flexible Metal-Organic Frameworks with Intrinsic Thermal Management. Nature 2015, 527, 357-361.

(13) Babaei, H.; McGaughey, A. J. H.; Wilmer, C. E. Effect of Pore Size and Shape on the Thermal Conductivity of Metal-Organic Frameworks. Chem. Sci. 2017, 8, 583-589.

(14) Ming, Y.; Purewal, J.; Liu, D.; Sudik, A.; Xu, C.; Yang, J.; Veenstra, M.; Rhodes, K.; Soltis, R.; Warner, J.; Gaab, M.; Müller, U.; Siegel, D. J. Thermophysical Properties of MOF-5 Powders. Microporous Mesoporous Mater. 2014, 185, 235-244.

(15) Makal, T. A.; Li, J.-R.; Lu, W.; Zhou, H.-C. Methane Storage in Advanced Porous Materials. Chem. Soc. Rev. 2012, 41, 7761-7779.

(16) Mason, J. A.; Veenstra, M.; Long, J. R. Evaluating MetalOrganic Frameworks for Natural Gas Storage. Chem. Sci. 2014, 5, 32 51.

(17) Li, B.; Wen, H.-M.; Zhou, W.; Xu, J. Q.; Chen, B. Porous Metal-Organic Frameworks: Promising Materials for Methane Storage. Chem 2016, 1, 557-580. 
(18) Chang, K. J.; Talu, O. Behavior and Performance of Adsorptive Natural Gas Storage Cylinders During Discharge. Appl. Therm. Eng. 1996, 16, 359-374.

(19) Beckner, M.; Dailly, A. A Pilot Study of Activated Carbon and Metal-Organic Frameworks for Methane Storage. Appl. Energy 2016, $162,506-514$.

(20) Mason, J. A.; Sumida, K.; Herm, Z. R.; Krishna, R.; Long, J. R. Evaluating Metal-Organic Frameworks for Post-Combustion Carbon Dioxide Capture via Temperature Swing Adsorption. Energy Environ. Sci. 2011, 4, 3030-3040.

(21) Trickett, C. A.; Helal, A.; Al-Maythalony, B. A.; Yamani, Z. H.; Cordova, K. E.; Yaghi, O. M. The Chemistry of Metal-Organic Frameworks for $\mathrm{CO}_{2}$ Capture, Regeneration and Conversion. Nat. Rev. Mater. 2017, 2, No. 17045.

(22) Schoedel, A.; Ji, Z.; Yaghi, O. M. The Role of Metal-Organic Frameworks in a Carbon-Neutral Energy Cycle. Nat. Energy 2016, 1, No. 16034.

(23) Chakraborty, A.; Kumar, S. Thermal Management and Desorption Modeling of a Cryo-Adsorbent Hydrogen Storage System. Int. J. Hydrogen Energy 2013, 38, 3973-3986.

(24) Xiao, J.; Hu, M.; Bénard, P.; Chahine, R. Simulation of Hydrogen Storage Tank Packed with Metal-Organic Framework. Int. J. Hydrogen Energy 2013, 38, 13000-13010.

(25) Wilmer, C. E.; Leaf, M.; Lee, C. Y.; Farha, O. K.; Hauser, B. G.; Hupp, J. T.; Snurr, R. Q. Large-Scale Screening of Hypothetical Metal-Organic Frameworks. Nat. Chem. 2012, 4, 83-89.

(26) Moghadam, P. Z.; Rogge, S. M. J.; Li, A.; Chow, C.-M.; Wieme, J.; Moharrami, N.; Aragones-Anglada, M.; Conduit, G.; GomezGualdron, D. A.; Van Speybroeck, V.; Fairen-Jimenez, D. StructureMechanical Stability Relations of Metal-Organic Frameworks via Machine Learning. Matter 2019, 1, 219-234.

(27) Sezginel, K. B.; Asinger, P. A.; Babaei, H.; Wilmer, C. E. Thermal Transport in Interpenetrated Metal-Organic Frameworks. Chem. Mater. 2018, 30, 2281-2286.

(28) Dubbeldam, D.; Walton, K. S.; Ellis, D. E.; Snurr, R. Q. Exceptional Negative Thermal Expansion in Isoreticular MetalOrganic Frameworks. Angew. Chem., Int. Ed. 2007, 46, 4496-4499.

(29) Alezi, D.; Belmabkhout, Y.; Suyetin, M.; Bhatt, P. M.; Weselinski, L. J.; Solovyeva, V.; Adil, K.; Spanopoulos, I.; Trikalitis, P. N.; Emwas, A.-H.; Eddaoudi, M. MOF Crystal Chemistry Paving the Way to Gas Storage Needs: Aluminum-Based soc-MOF for $\mathrm{CH}_{4}$, $\mathrm{O}_{2}$, and $\mathrm{CO}_{2}$ Storage. J. Am. Chem. Soc. 2015, 137, 13308-13318.

(30) Chui, S. S.-Y.; Lo, S. M.-F.; Charmant, J. P. H.; Orpen, G. A.; Williams, I. D. A Chemically Functionalizable Nanoporous Material $\left[\mathrm{Cu}_{3}(\mathrm{TMA})_{2}\left(\mathrm{H}_{2} \mathrm{O}\right)_{3}\right]_{n}$. Science 1999, 283, 1148-1150.

(31) Chen, B.; Ockwig, N. W.; Millward, A. R.; Contreras, D. S.; Yaghi, O. M. High $\mathrm{H}_{2}$ Adsorption in a Microporous Metal-Organic Framework with Open Metal Sites. Angew. Chem., Int. Ed. 2005, 44, $4745-4749$.

(32) Lin, X.; Jia, J.; Zhao, X.; Thomas, M.; Blake, A. J.; Walker, G. S.; Champness, N. R.; Hubberstey, P.; Schröder, M. High $\mathrm{H}_{2}$ Adsorption by Coordination-Framework Materials. Angew. Chem., Int. Ed. 2006, $45,7358-7364$.

(33) Cavka, J. H.; Jakobsen, S.; Olsbye, U.; Guillou, N.; Lamberti, C.; Bordiga, S.; Lillerud, K. P. A New Zirconium Inorganic Building Brick Forming Metal Organic Frameworks with Exceptional Stability. J. Am. Chem. Soc. 2008, 130, 13850-13851.

(34) Li, H.; Eddaoudi, M.; O’Keeffe, M.; Yaghi, O. M. Design and Synthesis of an Exceptionally Stable and Highly Porous MetalOrganic Framework. Nature 1999, 402, 276-279.

(35) Chae, H. K.; Siberio-Pérez, D. Y.; Kim, J.; Go, Y. B.; Eddaoudi, M.; Matzger, A. J.; O’Keeffe, M.; Yaghi, O. M. A Route to High Surface Area, Porosity and Inclusion of Large Molecules in Crystals. Nature 2004, 427, 523-527.

(36) Koh, K.; Wong-Foy, A. G.; Matzger, A. J. A Crystalline Mesoporous Coordination Copolymer with High Microporosity. Angew. Chem., Int. Ed. 2008, 47, 677-680.
(37) Dubbeldam, D.; Calero, S.; Vlugt, T. J. H. iRASPA: GPUAccelerated Visualization Software for Materials Scientists. Mol. Simul. 2018, 44, 653-676.

(38) Huang, B. L.; Ni, Z.; Millward, A.; McGaughey, A. J. H.; Uher, C.; Kaviany, M.; Yaghi, O. M. Thermal Conductivity of a MetalOrganic Framework (MOF-5): Part II. Measurement. Int. J. Heat Mass Transfer 2007, 50, 405-411.

(39) Mu, B.; Walton, K. S. Thermal Analysis and Heat Capacity Study of Metal-Organic Frameworks. J. Phys. Chem. C 2011, 115, 22748-22754.

(40) Kloutse, F. A.; Zacharia, R.; Cossement, D.; Chahine, R. Specific Heat Capacities of MOF-5, Cu-BTC, Fe-BTC, MOF-177 and MIL-53(Al) over Wide Temperature Ranges: Measurements and Application of Empirical Group Contribution Method. Microporous Mesoporous Mater. 2015, 217, 1-5.

(41) Lock, N.; Wu, Y.; Christensen, M.; Cameron, L. J.; Peterson, V. K.; Bridgeman, A. J.; Kepert, C. J.; Iversen, B. B. Elucidating Negative Thermal Expansion in MOF-5. J. Phys. Chem. C 2010, 114, 1618116186.

(42) Wu, Y.; Kobayashi, A.; Halder, G. J.; Peterson, V. K.; Chapman, K. W.; Lock, N.; Southon, P. D.; Kepert, C. J. Negative Thermal Expansion in the Metal-Organic Framework Material $\mathrm{Cu}_{3}(1,3,5-$ benzenetricarboxylate) $)_{2}$. Angew. Chem., Int. Ed. 2008, 47, 8929-8932.

(43) Erickson, K. J.; Léonard, F.; Stavila, V.; Foster, M. E.; Spataru, C. D.; Jones, R. E.; Foley, B. M.; Hopkins, P. E.; Allendorf, M. D.; Talin, A. A. Thin Film Thermoelectric Metal-Organic Framework with High Seebeck Coefficient and Low Thermal Conductivity. Adv. Mater. 2015, 27, 3453-3459.

(44) Huang, J.; Xia, X.; Hu, X.; Li, S.; Liu, K. A General Method for Measuring the Thermal Conductivity of MOF Crystals. Int. J. Heat Mass Transfer 2019, 138, 11-16.

(45) Eddaoudi, M.; Kim, J.; Rosi, N.; Vodak, D.; Wachter, J.; O’Keeffe, M.; Yaghi, O. M. Systematic Design of Pore Size and Functionality in Isoreticular MOFs and Their Application in Methane Storage. Science 2002, 295, 469-472.

(46) Vanduyfhuys, L.; Vandenbrande, S.; Verstraelen, T.; Schmid, R.; Waroquier, M.; Van Speybroeck, V. QuickFF: A Program for a Quick and Easy Derivation of Force Fields for Metal-Organic Frameworks from $\mathrm{Ab}$ Initio Input. J. Comput. Chem. 2015, 36, 1015-1027.

(47) Vanduyfhuys, L.; Vandenbrande, S.; Wieme, J.; Waroquier, M.; Verstraelen, T.; Van Speybroeck, V. Extension of the QuickFF Force Field Protocol for an Improved Accuracy of Structural, Vibrational, Mechanical and Thermal Properties of Metal-Organic Frameworks. J. Comput. Chem. 2018, 39, 999-1011.

(48) Lii, J. H.; Allinger, N. L. Molecular Mechanics. The MM3 Force Field for Hydrocarbons. 3. The van der Waals Potentials and Crystal Data for Aliphatic and Aromatic Hydrocarbons. J. Am. Chem. Soc. 1989, 111, 8576-8582.

(49) Frisch, M. J.; Trucks, G. W.; Schlegel, H. B.; Scuseria, G. E.; Robb, M. A.; Cheeseman, J. R.; Scalmani, G.; Barone, V.; Petersson, G. A.; Nakatsuji, H.; Li, X.; Caricato, M.; Marenich, A. V.; Bloino, J.; Janesko, B. G.; Gomperts, R.; Mennucci, B.; Hratchian, H. P.; Ortiz, J. V.; Izmaylov, A. F.; Sonnenberg, J. L.; Williams-Young, D.; Ding, F.; Lipparini, F.; Egidi, F.; Goings, J.; Peng, B.; Petrone, A.; Henderson, T.; Ranasinghe, D.; Zakrzewski, V. G.; Gao, J.; Rega, N.; Zheng, G.; Liang, W.; Hada, M.; Ehara, M.; Toyota, K.; Fukuda, R.; Hasegawa, J.; Ishida, M.; Nakajima, T.; Honda, Y.; Kitao, O.; Nakai, H.; Vreven, T.; Throssell, K., Montgomery, J. A., Jr.; Peralta, J. E.; Ogliaro, F.; Bearpark, M. J.; Heyd, J. J.; Brothers, E. N.; Kudin, K. N.; Staroverov, V. N.; Keith, T. A.; Kobayashi, R.; Normand, J.; Raghavachari, K.; Rendell, A. P.; Burant, J. C.; Iyengar, S. S.; Tomasi, J.; Cossi, M.; Millam, J. M.; Klene, M.; Adamo, C.; Cammi, R.; Ochterski, J. W.; Martin, R. L.; Morokuma, K.; Farkas, O.; Foresman, J. B.; Fox, D. J. Gaussian 16, revision E.01; Gaussian Inc.: Wallingford, CT, 2016.

(50) Becke, A. D. Density-Functional Thermochemistry. III. The Role of Exact Exchange. J. Chem. Phys. 1993, 98, 5648-5652. 
(51) Krishnan, R.; Binkley, J. S.; Seeger, R.; Pople, J. A. SelfConsistent Molecular Orbital Methods. 20. Basis Set for Correlated Wave-Functions. J. Chem. Phys. 1980, 72, 650-654.

(52) Hay, P. J.; Wadt, W. R. Ab Initio Effective Core Potentials for Molecular Calculations. Potentials for the Transition Metal Atoms Sc to Hg. J. Chem. Phys. 1985, 82, No. 270.

(53) Verstraelen, T.; Vandenbrande, S.; Heidar-Zadeh, F.; Vanduyfhuys, L.; Van Speybroeck, V.; Waroquier, M.; Ayers, P. W. Minimal Basis Iterative Stockholder: Atoms in Molecules for ForceField Development. J. Chem. Theory Comput. 2016, 12, 3894-3912.

(54) Perdew, J. P.; Burke, K.; Ernzerhof, M. Generalized Gradient Approximation Made Simple. Phys. Rev. Lett. 1996, 77, 3865-3868.

(55) Mortensen, J. J.; Hansen, L. B.; Jacobsen, K. W. Real-Space Grid Implementation of the Projector Augmented Wave Method. Phys. Rev. B 2005, 71, No. 035109.

(56) Kapil, V.; Behler, J.; Ceriotti, M. High Order Path Integrals Made Easy. J. Chem. Phys. 2016, 145, No. 234103.

(57) Kapil, V.; Wieme, J.; Vandenbrande, S.; Lamaire, A.; Van Speybroeck, V.; Ceriotti, M. Modeling the Structural and Thermal Properties of Loaded Metal-Organic Frameworks. An Interplay of Quantum and Anharmonic Fluctuations. J. Chem. Theory Comput. 2019, 15, 3237-3249.

(58) Markland, T. M.; Ceriotti, M. Nuclear Quantum Effects Enter the Mainstream. Nat. Rev. Chem. 2018, 2, No. 0109.

(59) Kapil, V.; VandeVondele, J.; Ceriotti, M. Accurate Molecular Dynamics and Nuclear Quantum Effects at Low Cost by Multiple Steps in Real and Imaginary Time: Using Density Functional Theory to Accelerate Wavefunction Methods. J. Chem. Phys. 2016, 144, No. 054111.

(60) Ceriotti, M.; More, J.; Manolopoulos, D. E. i-PI: A Python Interface for Ab Initio Path Integral Molecular Dynamics Simulations. Comput. Phys. Commun. 2014, 185, 1019-1026.

(61) Kapil, V.; Rossi, M.; Marsalek, O.; Petraglia, R.; Litman, Y.; Spura, T.; Cheng, B.; Cuzzocrea, A.; Meißner, R. H.; Wilkins, D. M.; Juda, P.; Bienvenue, S. P.; Fang, W.; Kessler, J.; Poltavsky, I.; Vandenbrande, S.; Wieme, J.; Corminboeuf, C.; Kühne, T. D.; Manolopoulos, D. E.; Markland, T. E.; Richardson, J. O.; Tkatchenko, A.; Tribello, G. A.; Speybroeck, V. V.; Ceriotti, M.; et al. i-PI 2.0: A Universal Force Engine for Advanced Molecular Simulations. Comput. Phys. Commun. 2019, 236, 214-223.

(62) Verstraelen, T.; Vanduyfhuys, L.; Vandenbrande, S.; Rogge, S. M. J.Yaff 1.4.2., 2017. http://molmod.github.io/yaff/index.html.

(63) Plimpton, S. Fast Parallel Algorithms for Short-Range Molecular Dynamics. J. Comput. Phys. 1995, 117, 1-19.

(64) Lamaire, A.; Wieme, J.; Rogge, S. M. J.; Waroquier, M.; Van Speybroeck, V. On the Importance of Anharmonicities and Nuclear Quantum Effects in Modelling the Structural Properties and Thermal Expansion in MOF-5. J. Chem. Phys 2019, 150, No. 094503.

(65) Green, M. S. Markoff Random Processes and the Statistical Mechanics of Time-Dependent Phenomena. II. Irreversible Processes in Fluids. J. Chem. Phys. 1954, 22, 398-413.

(66) Kubo, R. Statistical-Mechanical Theory of Irreversible Processes. I. General Theory and Simple Applications to Magnetic and Conduction Problems. J. Phys. Soc. Jpn. 1957, 12, 570-586.

(67) Rogge, S. M. J.; Vanduyfhuys, L.; Ghysels, A.; Waroquier, M.; Verstraelen, T.; Maurin, G.; Van Speybroeck, V. A Comparison of Barostats for the Mechanical Characterization of Metal-Organic Frameworks. J. Chem. Theory Comput. 2015, 11, 5583-5597.

(68) Schelling, P. K.; Phillpot, S. R.; Keblinski, P. Comparison of Atomic-Level Simulation Methods for Computing Thermal Conductivity. Phys. Rev. B 2002, 65, No. 144306.

(69) McGaughey, A. J. H.; Kaviany, M. Thermal Conductivity Decomposition and Analysis Using Molecular Dynamics Simulations. Part II. Complex Silica Structures. Int. J. Heat Mass Transfer 2004, 47, $1799-1816$.

(70) Huang, B. L.; McGaughey, A. J. H.; Kaviany, M. Thermal Conductivity of Metal-Organic Framework 5 (MOF-5): Part I. Molecular Dynamics Simulations. Int. J. Heat Mass Transfer 2007, 50, 393-404.
(71) Dubbeldam, D.; Calero, S.; Ellis, D. E.; Snurr, R. Q. RASPA: Molecular Simulation Software for Adsorption and Diffusion in Flexible Nanoporous Materials. Mol. Simul. 2016, 42, 81-101.

(72) Willems, T. F.; Rycroft, C. H.; Kazi, M.; Meza, J. C.; Haranczyk, M. Algorithms and Tools for High-Throughput Geometry-Based Analysis of Crystalline Porous Materials. Microporous Mesoporous Mater. 2012, 149, 134-141.

(73) Cui, B.; Audu, C. O.; Liao, Y.; Nguyen, S. T.; Farha, O. K.; Hupp, J. T.; Grayson, M. Thermal Conductivity of ZIF-8 Thin-Film Under Ambient Gas Pressure. ACS Appl. Mater. Interfaces 2017, 9, 28139-28143.

(74) Zhang, X.; Jiang, J. Thermal Conductivity of Zeolitic Imidazolate Framework-8: A Molecular Simulation Study. J. Phys. Chem. C 2013, 36, 18441-18447.

(75) Han, L.; Budge, M.; Greaney, A. P. Relationship between Thermal Conductivity and Framework Architecture. Comput. Mater. Sci. 2014, 94, 292-297.

(76) Kumar, K. V.; Preuss, K.; Titirici, M.-M.; Rodriguez-Reinoso, F. Nanoporous Materials for the Onboard Storage of Natural Gas. Chem. Rev. 2017, 117, 1796-1825.

(77) de Lange, M. F.; Verouden, K. J. F. M.; Vlugt, T. J. H.; Gascon, J.; Kapteijn, F. Adsorption-Driven Heat Pumps: The Potential of Metal-Organic Frameworks. Chem. Rev. 2015, 115, 12205-12250.

(78) Stassen, I.; Burtch, N. C.; Talin, A.; Falcaro, P.; Allendorf, M.; Ameloot, R. An Updated Roadmap for the Integration of MetalOrganic Frameworks with Electronic Devices and Chemical Sensors. Chem. Soc. Rev. 2017, 46, 3185-3241.

(79) Coates, C. S.; Goodwin, A. How to Quantify Isotropic Negative Thermal Expansion: Magnitude, Range, or Both? Mater. Horiz. 2019, 6, 211-218.

(80) Lock, N.; Christensen, M.; Wu, Y.; Peterson, V. K.; Thomsen, M. K.; Plitz, R. O.; Ramirez-Cuesta, A. J.; McIntyre, G. J.; Norén, K.; Kutteh, R.; Kepert, C. J.; Kearley, G. J.; Iversen, B. B. Scrutinizing Negative Thermal Expansion in MOF-5 by Scattering Techniques and Ab Initio Calculations. Dalton Trans. 2013, 42, 1996-2007.

(81) Cliffe, M. J.; Hill, J. A.; Murray, C. A.; Coudert, F.-X.; Goodwin, A. L. Defect-Dependent Colossal Negative Thermal Expansion in UiO-66(Hf) Metal-Organic Frameworks. Phys. Chem. Chem. Phys. 2015, 17, 11586-11592.

(82) Tan, J.-C.; Cheetham, A. K. Mechanical Properties of Hybrid Inorganic-Organic Framework Materials: Establishing Fundamental Structure-Property Relationships. Chem. Soc. Rev. 2011, 40, 10591080.

(83) Nandasiri, M. I.; Jambovane, S. R.; McGrail, P. B.; Schaef, H. T.; Nune, S. K. Adsorption, Separation, and Catalytic Properties of Densified Metal-Organic Frameworks. Coord. Chem. Rev. 2016, 311, $38-52$.

(84) Tian, T.; Zeng, Z.; Vulpe, D.; Casco, M. E.; Divitini, G.; Midgley, P. A.; Silvestre-Albero, J.; Tan, J.-C.; Moghadam, P. Z.; Fairen-Jimenez, D. A Sol-Gel Monolithic Metal-Organic Framework with Enhanced Methane Uptake. Nat. Mater. 2018, 17, 174-179.

(85) Purewal, J. J.; Liu, D.; Yang, J.; Sudik, A.; Siegel, D. J.; Maurer, S.; Müller, U. Increased Volumetric Hydrogen Uptake of MOF-5 by Powder Densification. Int. J. Hydrogen Energy 2012, 37, 2723-2727.

(86) Purewal, J.; Liu, D.; Sudik, A.; Veenstra, M.; Yang, J.; Maurer, S.; Müller, U.; Siegel, D. J. Improved Hydrogen Storage and Thermal Conductivity in High-Density MOF-5 Composites. J. Phys. Chem. C 2012, 116, 20199-20212.

(87) Liu, D.; Purewal, J. J.; Yang, J.; Sudik, A.; Maurer, S.; Müller, U.; Ni, J.; Siegel, D. J. MOF-5 Composites Exhibiting Improved Thermal Conductivity. Int. J. Hydrogen Energy 2012, 37, 6109-6117.

(88) Jeremias, F.; Henninger, S. K.; Janiak, C. High Performance Metal-Organic Framework Coatings Obtained via Thermal Gradient Synthesis. Chem. Commun. 2012, 48, 9708-9710.

(89) He, Y.; Zhou, W.; Qian, G.; Chen, B. Methane Storage in Metal-Organic Frameworks. Chem. Soc. Rev. 2014, 43, 5657-5678.

(90) Ren, J.; Langmi, H. W.; North, B. C.; Mathe, M. Review on Processing of Metal-Organic Framework (MOF) Materials towards 
System Integration for Hydrogen Storage. Int. J. Energy Res. 2015, 39, 607-620.

(91) Babaei, H.; Wilmer, C. E. Mechanisms of Heat Transfer in Porous Crystals Containing Adsorbed Gases: Applications to MetalOrganic Frameworks. Phys. Rev. Lett. 2016, 116, No. 025902.

(92) Balestra, S. R. G.; Bueno-Perez, R.; Hamad, S.; Dubbeldam, D.; Ruiz-Salvador, A. R.; Calero, S. Controlling Thermal Expansion: AMetal-Organic Frameworks Route. Chem. Mater. 2016, 28, 82968304.

(93) Auckett, J. E.; Barkhordarian, A. A.; Ogilvie, S. H.; Duyker, S. G.; Chevreau, H.; Peterson, V. K.; Kepert, C. J. Continuous Negativeto-Positive Tuning of Thermal Expansion Achieved by Controlled Gas Sorption in Porous Coordination Frameworks. Nat. Commun. 2018, 9, No. 4873.

(94) Horike, S.; Shimomura, S.; Kitagawa, S. Soft Porous Crystals. Nat. Chem. 2009, 1, 695-704.

(95) Vanduyfhuys, L.; Rogge, S. M. J.; Wieme, J.; Vandenbrande, S.; Maurin, G.; Waroquier, M.; Van Speybroeck, V. Thermodynamic Insight into Stimuli-Responsive Behaviour of Soft Porous Crystals. Nat. Commun. 2018, 9, No. 204.

(96) Babaei, H.; McGaughey, A. J. H. Transient Mass and Thermal Transport during Methane Adsorption into the Metal-Organic Framework HKUST-1. ACS Appl. Mater. Interfaces 2018, 10, 24002406. 